\title{
Occult gunshot injury of the temporal bone
}

\author{
U M Geary, D A Ritchie, L C Luke
}

\begin{abstract}
Increasing firearms violence has produced much public disquiet in recent months and Liverpool has seen a particularly well publicised spate of shootings. This is a case report of an initially occult intracranial injury which illustrates the unpredictable nature of missile trauma and the importance of computerised tomography in all cases of gunshot injury to the head.

(F Accid Emerg Med 1997;14:185-186)
\end{abstract}

Keywords: intracranial injury; gunshot wound; temporal bone

Royal Liverpool

University Hospital, Liverpool:

Department of

Accident and

Emergency Medicine

U M Geary

L C Luke

Department of

Radiology

D A Ritchie

Correspondence to:

Dr U M Geary, Departmen of Accident and Emergency Medicine, Royal Liverpool University Hospital, Prescot Street, Liverpool L7 8XP.

Accepted for publication 27 January 1997

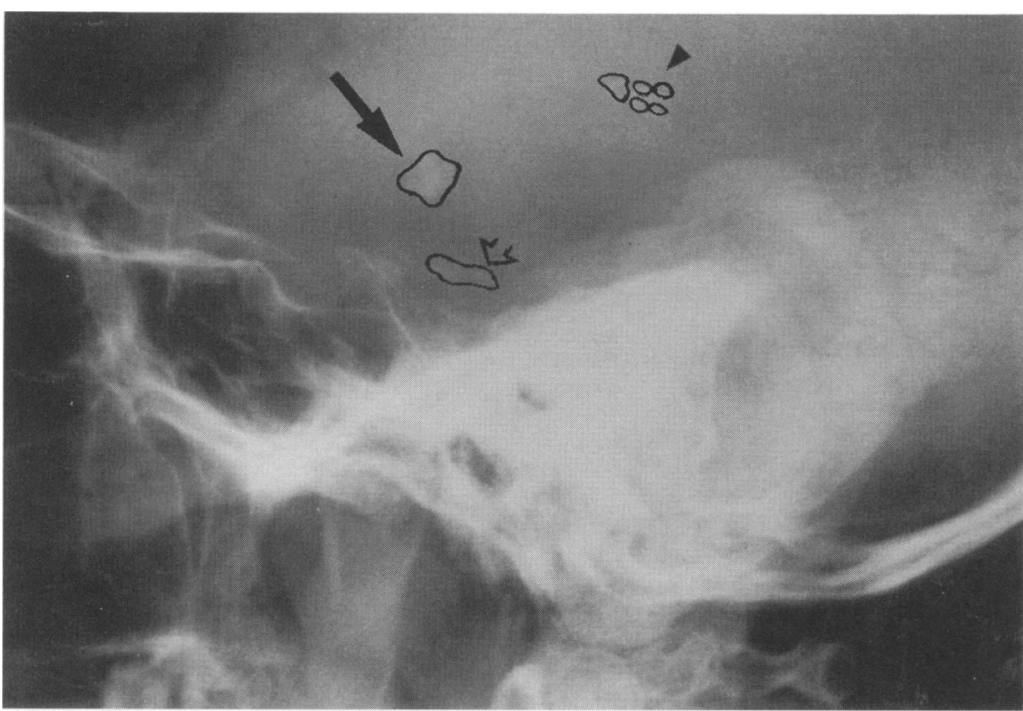

Figure 1 Lateral skull radiograph. Irregular $8 \mathrm{~mm}$ calcific opacity (straight arrow) and further smaller ill defined calcific opacities (open arrow) over the temporal region, only noted on lateral film, were initially thought to be of no clinical significance. Further imaging showed they represented bone fragments lying within the right temporal fossa. Note the physiological calcifications of the pineal gland and habenular commisure (arrowhead). len and there was right sided epistaxis. Swelling of the uvula was the only abnormality detected on intraoral examination. No evidence of cranial or peripheral neuropathology was found. Skull radiography was performed but no metal fragments or vault fractures were seen. The calcific opacities over the temporal region on the lateral view were not thought to be of any clinical significance (fig 1). Half an hour after the patient's arrival the police reported that they had discovered a $9 \mathrm{~mm}$ bullet at the scene and, in the absence of clinically manifest intracranial injury or discernible exit wound, it was concluded that the patient had received a glancing bullet wound to the head. Accordingly, he was admitted for routine neurological monitoring, further facial radiographs, and maxillo-facial and ear, nose and throat (ENT) surgical consultation. Five hours after the patient's admission to the short stay observation ward, he suffered a self limiting generalised seizure.

Urgent computerised tomography (CT) of the brain (fig 2) showed an acute comminuted intra-articular fracture of the antero-inferior portion of the right temporal bone involving the mandibular fossa and external auditory canal. Further fractures of the right mandibular head and medial and lateral walls of the right maxillary antrum were also revealed. There was an $8 \mathrm{~mm}$ bony fragment within the right temporal lobe surrounded by some oedema and haemorrhage. Haemorrhage was also noted in the subarachnoid spaces of the right sylvian fissure and tentorium cerebelli, but there was no retained foreign body. A small pneumocephalus was demonstrated.

Following consultation with the maxillofacial and ENT surgeons, an expectant management policy was agreed. Anti-epileptic treatment was started, together with antibiotic and anti-tetanus prophylaxis. The patient had no further seizures while in hospital and a CSF leak from the right ear resolved after five days. Repeat CT scanning excluded further intracranial pathology and the patient was discharged from hospital after one week. His only remaining neuropathology was continued deafness.

\section{Discussion}

The wound ballistics in this case were remarkable. The CT images show that the bullet entered through the post-auricular wound and after striking the temporal bone was deflected anteriorly, travelling through the infratemporal fossa, shattering the articular process of the mandible, and then entering the right maxillary sinus. The bullet probably made its exit through the nose. Missiles penetrating a 


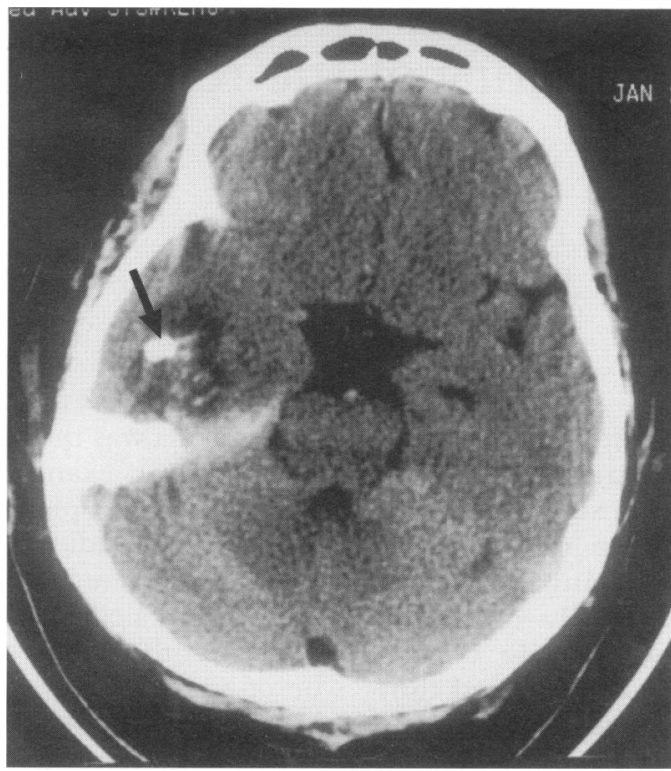

Figure 2 Computerised tomography of the brain (brain settings); $8 \mathrm{~mm}$ bony fragment (arrow) lying within the right temporal lobe with some surrounding oedema/haemorrhage.

bony area covered by only a thin layer of skin may produce a split laceration rather than a characteristic bullet entry wound. ${ }^{1}$ Fragmentation is common in temporal bone gunshot injury, as the energy of the bullet's velocity is transmitted to the relatively resistant hard bone. Associated mandibular and facial fractures have been described before. ${ }^{2}$ Neurovascular injury is responsible for the high mortality ( $52 \%$ in one American series ${ }^{2}$ ) and morbidity associated with temporal gunshot injury. Hearing loss, which may be either conductive or sensory, is common in survivors. Cholesteatoma may result from implantation of the skin in the mastoid or cranial fossa Facial nerve damage is characteristic of gunshot injury to the temporal bone; other cranial nerves may also be damaged. The anterior part of the temporal lobe is not "neurologically eloquent", ${ }^{3}$ so isolated brain injury in this region may be missed on cursory examination Subdural haematoma has been reported in cases of non-penetrating "glancing" bullet injuries to the temporal region. ${ }^{4}$ The incidence of seizures is increased to about $40 \%{ }^{5} \mathrm{com}$ pared with $3-5 \%$ in unselected (mostly closed) head injuries. ${ }^{6}$ Psychosocial morbidity should also be anticipated in patients surviving serious head injury.

In this case, the calcific opacities noted on the lateral skull radiograph were initially misin- terpreted as insignificant. However, when assessing such lesions one should consider the possibilities of intracranial and extracranial foreign bodies and physiological and pathological intracranial calcifications. Foreign bodies associated with gunshot injuries are likely to be of metallic density. While the lower ill defined calcific opacities over the temporal region may have represented physiological calcification within the petro-clinoid ligaments, the larger opacity lying superiorly does not correspond to any physiological calcification and therefore must have represented a pathological lesion. Although there are numerous causes of pathological intracranial calcifications, the clinical presentation should have raised the possibility of bony fragments from a gunshot injury.

Computerised tomography is the imaging method of choice in patients with penetrating cranial trauma. ${ }^{7}$ It is mandatory where penetrating or glancing bullet injuries are suspected. Axial and coronal imaging delineates complicated injuries of the inner ear or craniofacial skeleton. Plain skull $x$ rays may be useful in demonstrating the overall picture of missile distribution and fragmentation. Angiography is not routinely performed but may be indicated by clinical findings or CT appearances. Imaging should also include radiography of the cervical spine to exclude further injury.

The management of patients of gunshot injury to the head is based upon resuscitation along standard advanced trauma life support (ATLS) guidelines, routine comuterised tomography in all cases and close liaison with radiological and neurosurgical colleagues. Prophylactic antibiotics are currently recommended for such injuries. ${ }^{8}$

1 Stone IC, Petty CS. Interpretation of unusual wounds caused by firearms. J Forensic Sci 1991;36:736-40.

2 Stack BC, Farrior JB. Missile injuries to the temporal bone. South Med J 1995;88:72-8.

3 Rosenwasser RH, Andrews DW, Jimenez DF. Penetrating craniocerebral trauma. Surg Clin North Am 1991;71:305-

4 Hadas N, Schiffer J, Rogev M, Shperber Y. Tangential low velocity missile wound of the head with acute subdural haematoma: case report. J Trauma 1990;30:358-9.

5 Annagers JF, Grabow JD, Groover RJ, Laws AR, Elveback LR, Kurland LT. Seizures after head trauma : a population study. Neurology 1980;30:683-9.

6 Pagni DA. Post traumatic epilepsy, incidence and prophylaxis. Acta Neurochir 1990;50(suppl):8-47.

7 Phillips CD. Emergent radiologic evaluation of the gunshot wound victim. Radiol Clin North Am 1992;30:307-24.

8 Dunn LT, Foy PM. Anti-convulsant and antibiotic prophylaxis in head injury. Ann R Coll Surg Engl 1994;76:147-9. 